\title{
BLOG Y TWITTER, LA COMBINACIÓN PERFECTA DEL COMUNICADOR DIGITAL: LOS CASOS DE ESCOLAR.NET, EL COMIDISTA Y MII MESA COJEA
}

\section{Blog and Twitter, the perfect combination for a digital communicator: The cases of Escolar.net, El comidista and Mi mesa cojea}

\section{Íñigo Marauri-Castillo, María-José Cantalapiedra-González y Carmen Álvarez-Fernández}

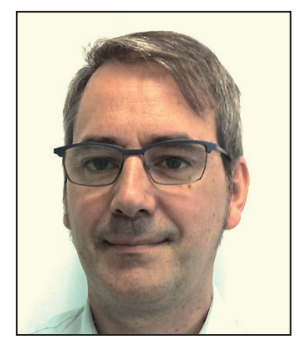

íñigo Marauri-Castillo es profesor ayudante doctor del Departamento de Periodismo I/ de la Universidad del País Vasco (UPV/EHU). Tras 15 años de experiencia profesional en los diarios El correo, El país y la revista Consumer Eroski, desde 2011 su labor se ha centrado en la docencia y la investigación. Sus principales líneas de estudio son el tratamiento de los sucesos y el impacto de internet en la comunicación corporativa y en el periodismo de servicio.

https://orcid.org/0000-0003-0883-8003

Universidad del País Vasco Facultad de Ciencias Sociales y de la Comunicación Campus de Bizkaia. Barrio Sarriena, s/n. 48940 Leioa, España

inigo.marauri@ehu.es

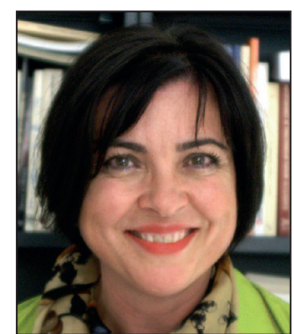

María-José Cantalapiedra-González es profesora titular del Departamento de Periodismo I/ de la Universidad del País Vasco (UPV/EHU). Entre sus líneas de investigación se encuentra la evolución de los géneros periodísticos, su transposición a internet, así como el impacto y las posibilidades que brindan las nuevas tecnologías a los profesionales de la información y de la comunicación corporativa. Ha dirigido una quincena de tesis doctorales, doce de ellas ya defendidas. https://orcid.org/0000-0003-4961-2326

Universidad del País Vasco, Facultad de Ciencias Sociales y de la Comunicación Campus de Bizkaia, Barrio Sarriena, s/n. 48940 Leioa, España mariajose.cantalapiedra@ehu.es



Carmen Álvarez-Fernández es master de Investigación en Comunicación Social por la Universidad del País Vasco (UPV/EHU). Periodista y profesional digital con 2 años de experiencia en comunicación corporativa en empresas. Es responsable del departamento de comunicación y marketing de Quoters, un software online de presupuestos. En Quoters lleva a cabo estrategias de inbound marketing, donde la elaboración de contenido en blogs y redes sociales es el eje fundamental de las acciones.

https://orcid.org/0000-0002-2280-1728

Cloud Man Lab Acera de Recoletos, 6, entr. 47004 Valladolid, España carmenaf.91@gmail.com

\section{Resumen}

Marca personal y periodismo definen el presente de los profesionales de la información en Internet. El aprovechamiento de las posibilidades que brindan Twitter y los blogs para mostrar y viralizar un contenido puede lanzar la carrera laboral de su autor. Este artículo abre una línea de investigación sobre una realidad incipiente e inexplorada del branding personal. Para ello, se han tomado como referencia tres casos: Ignacio Escolar (Escolar.net), Mikel López-Iturriaga (El comidista) y José-Antonio Pérez (Mi mesa cojea). Sus reflexiones y experiencias, complementadas con las de empleadores y expertos, permiten ofrecer una primera visión sobre las posibilidades y los límites del sistema.

\section{Palabras clave}

Periodismo; Blogs; Medios sociales; Redes sociales; Marca personal; Twitter; Periodistas; Comunicadores; Entrevistas; Escolar.net; El comidista; Mi mesa cojea. 


\begin{abstract}
Personal branding and journalism help define an information professionals' identity on the Internet. The benefits of Twitter and blogs can be leveraged to promote the author's career. This article aims to open a research line into the emerging reality of journalists' personal branding. Therefore, the cases of three journalists in Spain: Ignacio Escolar (Escolar.net), Mikel López-Iturriaga (El comidista), and José-Antonio Pérez (Mi mesa cojea) are studied. Their reflections and experiences, along with information from employers and experts, offers an early glimpse into the opportunities and boundaries of leveraging professional identity on the Internet.
\end{abstract}

\title{
Keywords
}

Journalism; Blogs; Social media; Social networks; Personal branding; Twitter; Journalists; Communicators; Interviews; Escolar.net; El comidista; Mi mesa cojea.

Marauri-Castillo, Îñigo; Cantalapiedra-González, María-José; Álvarez-Fernández, Carmen (2018). “Blog y Twitter, la combinación perfecta del comunicador digital: los casos de Escolar.net, El comidista y Mi mesa cojea". El profesional de la información, v. 27, n. 2, pp. 349-358.

https://doi.org/10.3145/epi.2018.mar.13

\section{Introducción}

Las redes sociales son parte relevante del ecosistema informativo en internet. Su identificación como redes de valor (Campos-Freire et al., 2016) para los medios de comunicación se ha trasladado también al plano individual y del mismo modo que transforman la actividad de las empresas, impactan en periodistas y comunicadores digitales (Brems et al., 2017, p. 443).

La combinación de redes sociales y plataformas de autopublicación de contenido permite a los profesionales de la comunicación conseguir reconocimiento, reputación y remuneración. La vinculación con una empresa informativa para crear y distribuir contenido ya no es una necesidad, sino una opción. Esta transformación multiplica su relevancia en un contexto en el que la reducción a escala global de redacciones y presupuestos es un hecho (Brems et al., 2017, p. 444; Hanusch; Bruns, 2017, p. 28). Esta realidad incide en las figuras laborales del periodismo. En el caso español, el Informe anual de la profesión periodística 2016 (APM, 2016) señala que el 27\% de los periodistas encuestados trabaja como autónomo, una tendencia creciente ya que el porcentaje en 2013 era del 15\%.

Entran en liza conceptos como la marca personal y la reputación online de profesionales que han logrado reconocimiento y empleo gracias a la calidad de sus contenidos y a su habilidad para viralizarlos. En España se han convertido en referencias de una alternativa de promoción profesional casos como los de:

- Jordi Pérez-Colomé, con su blog Obama world

- Manuel Moreno, con Trece bits

- José-Antonio Pérez, autor de Mi mesa cojea

- Mikel López-Iturriaga, El comidista

- Ignacio Escolar, creador de Escolar.net

Todos tienen una característica en común: han tenido como origen un blog al que han sumado al menos una cuenta de Twitter. Blog, Twitter, periodismo, marca personal y empleabilidad son palabras que se relacionan de manera creciente.

Desde una perspectiva cualitativa y exploratoria, se han seleccionado tres casos de éxito (Ignacio Escolar, Mikel
López-Iturriaga y José-Antonio Pérez) para una primera aproximación a un fenómeno que representa una alternativa al acceso del mercado de trabajo de la comunicación en general y del periodismo en particular.

Se han fijado los siguientes objetivos:

1. Perfilar la relevancia para un periodista de mantener un blog y una cuenta de Twitter activos como medio de obtener trabajo en el sector y conocer si en esta labor se incluye Facebook como recurso complementario.

2. Desglosar las funciones de un blog y una cuenta de Twitter como medios de creación de marca personal para un periodista y/o comunicador.

3. Identificar pautas de trabajo para un mejor aprovechamiento de las posibilidades que ofrecen el blog y Twitter como tarjetas de presentación de un periodista.

4. Esbozar las tendencias en las demandas que los principales medios online españoles, nativos digitales o no, establecen para los nuevos profesionales.

El cumplimiento de estos objetivos pretende confirmar las siguientes hipótesis:

1. Un blog y una cuenta de Twitter conforman el principal binomio para un periodista que desea dar a conocer su trabajo en internet. Su uso coordinado se ha convertido en un medio para lograr captar la atención de empleadores hacia sus contenidos, y con ello, conseguir ofertas de trabajo.

2. La combinación del aprovechamiento de las posibilidades de publicación y viralidad que brindan el blog y Twitter con unos contenidos de calidad permite construir la marca personal y la reputación online, que puede materializarse en ofertas laborales.

3. El blog se usa como plataforma de publicación de contenidos propios para cuya promoción se utiliza principalmente Twitter. El carácter menos abierto de Facebook hace que esta red, pese a contar con un mayor número de usuarios, ocupe un papel secundario.

4. Las posibilidades de encontrar trabajo crecen cuanto mayor es el número de seguidores de blogs y Twitter. 


\section{Twitter, blog y el periodismo adaptativo basado en el individuo}

Es complejo establecer los cambios que internet ha supuesto para la figura del periodista. Por un lado las competencias tradicionales no han cambiado en internet (Palomo, 2013) y representan aún la base de la demanda de empleo del sector periodístico (Bakker, 2014, Palomo; Palau-Sampio, 2016). Pero las herramientas de trabajo sí se han transformado, por lo que se incide en la necesidad de contar con la figura bautizada como "periodista adaptativo" (Palomo; Palau-Sampio, 2016).

El gran reto es cómo encajar la labor del periodista en un sistema con diferentes reglas y en cómo fijar rutinas adecuadas a las pautas marcadas por los cambios sociales y tecnológicos (Noguera-Vivo, 2013). Este contexto se asocia con el ambient journalism (Hermida, 2010), definido como un entorno social en el que la interacción entre ciudadanos y periodistas está transformando la labor periodística, en la que adquiere un mayor peso el proceso frente al producto. Una visión que encaja con la idea de que, en el periodismo, una habilidad esencial es "escuchar y cambiar" (Jarvis, 2014).

En esa adaptación, las redes sociales (Picard, 2014; 2015, Molyneux; Holton, 2015) y el blog asumen un rol protagonista como instrumentos para crear o potenciar la figura del profesional de la comunicación en general y del periodista en particular. Entre los medios sociales sobresale Twitter como herramienta fundamental para los periodistas (Rodríguez-Rubial; García-López, 2013, López-Meri, 2015). Tanto es así que se identifica como la red que simboliza el ambient journalism y lo que Noguera-Vivo (2013) define como periodismo centrado en el usuario. Se convierte en un lugar de encuentro y de mutuo aprendizaje entre profesionales del periodismo y periodistas amateur en el que la esencia colaborativa y horizontal marca su relación. Este contexto propicia la creación de una identidad pública para el periodista, y de manera paralela un proceso de co-creación de contenido. Esto contribuye a una conexión emocional con los consumidores (Molyneux; Holton, 2015), a los que se trata y se sienten tratados como una activa comunidad de participantes y no como un grupo estático de receptores.

La consecuencia obvia de este cambio es que a las tareas del periodista se han sumado la alimentación, la revisión y el cuidado de las redes sociales (Hermida, 2013). No obstante, el porcentaje que hace un uso activo y constante representa una pequeña aunque creciente fracción del conjunto de periodistas (Hedman; Djerf-Pierre, 2013).

En esta transformación hay dos referentes y guías:

- los propios periodistas;

- la comunidad de usuarios de las redes sociales.

La inmersión en estas últimas es la que permite a los periodistas aprender las normas, usos y costumbres de sus comunidades de usuarios (Lasorsa; Lewis; Holton, 2012).

El blog ofrece, junto con comentarios, análisis e información, una visión más personal de quien lo firma. Similar descripción es extrapolable a Twitter y a la twitter-retórica o lenguaje propio de Twitter (López-Meri; Casero-Ripollés,
2017), lo que representa un estadio que va más allá de la práctica habitual en las cuentas oficiales de los medios basada en repetir titulares. Esto explicaría que en Twitter la referencia periodística sean las personas y no las empresas, y que autores como Noguera-Vivo (2013, p. 101) recomienden que las empresas periodísticas tomen nota del uso de este canal por algunos blogueros, colaboradores, editores y corresponsales. Lo cierto es que Twitter abandera una transformación en la que los individuos pueden estar sustituyendo a las instituciones como unidad fundamental de la profesión periodística (Molyneux; Holton, 2015, p. 226) y en el que el yo pasa a ser una herramienta más del periodista (Brems et al., 2017, p. 456). La suma de los factores tecnológicos y de los efectos de una recesión económica que ha golpeado con especial virulencia a la profesión periodística hace que las organizaciones muestren signos de debilidad y las personas ganen poder e influencia. La idea del sujeto como marca ha entrado con fuerza en la descripción del periodismo en internet.

\section{Marca personal}

Pese a un creciente y patente interés por la vinculación entre la figura del periodista y el concepto de marca personal, la asociación de una y otro no ha tenido un reflejo extendido en la investigación académica. Los primeros estudios limitan la marca al ámbito organizacional, aunque a medida que crecen los trabajos acerca de Twitter se constata un giro hacia la marca personal del periodista.

El concepto de marca personal, en inglés personal branding, proviene de la bibliografía empresarial de Estados Unidos y fue creado a finales de los años noventa por Tom Peters. Éste escribió en agosto de 1997 en la revista Fast company un artículo emblemático en el que se establecía la filosofía básica del personal branding (Pérez-Ortega, 2012, p. 87). Habilidades, personalidad y singularidad definen una idea que se plasma en el caso de los periodistas en una combinación que busca un difícil equilibrio entre autopromoción, información, opinión, crítica y argumentación (Brems et al., 2017, López-Meri; Casero-Ripollés, 2016).

Pérez-Ortega (2012) evalúa el desarrollo de la marca personal en internet entre la profesión periodística. Entre las deficiencias que a su juicio los periodistas deben solventar para aprovechar su marca personal en internet, resalta:

- un mayor y mejor uso de Twitter y el blog;

- una mayor especialización que permita una mayor individualización;

- un mayor protagonismo del periodista frente al medio en que trabaja, protagonismo en el que debe colaborar el propio medio.

En los primeros trabajos que abordan el estudio de los objetivos con el que los periodistas utilizaban las redes sociales, la creación de marca personal aparecía en los últimos lugares, sensiblemente por debajo de la labor de apoyo a la marca del medio (Hedman; Djerf-Pierre, 2013), aunque se constataba asimismo que la preocupación por la marca personal era mayor en los periodistas que usaban de forma frecuente las redes sociales. En un estudio protagonizado por periodistas estadounidenses especializados en salud, los participantes aseguraban que la creación de marca se 
había convertido en una parte de su práctica diaria (Molyneux; Holton, 2015, p. 231).

Esta visión encaja con la descripción del uso de Twitter de los periodistas realizada por Swasey (2017) y con los motivos que explican, según Molyneux (2015), el interés de los periodistas por fomentar su propia marca: mejora de su trabajo, deseo de lograr una voz propia diferenciada del medio y narcisismo.

Los profesionales del periodismo usan cuatro estrategias según López-Meri y Casero-Ripollés (2017): posicionamiento, curación o recomendación de contenidos, personalización, y especialización. Estas estrategias son extensibles a la labor que se lleva a cabo en los blogs. En esa tarea, funciones que a priori y desde el plano teórico se han fijado como idóneas para Twitter, como la interactividad y el diálogo (Molyneux, 2015) con el resto de la sociedad, quedan relegadas a un segundo plano ante una constatable endogamia, al menos en el caso español (Arrabal-Sánchez; De-Aguilera-Moyano, 2016).

\section{Metodología}

\subsection{Tipo de investigación}

Se ha realizado una investigación cualitativa a partir de la entrevista en profundidad a doce profesionales del mundo de la comunicación, aglutinados en tres perfiles:

- casos de estudio;

- empleadores;

- expertos.

Se ha seguido una metodología cualitativa porque la intención de la investigación es interpretar y comprender la realidad de la profesión periodística a través del sentir de los entrevistados, es decir, desde su perspectiva, que es subjetiva (Taylor; Bogdan, 1992, Banister et al., 2004). Pasar de llamar a las personas de la investigación "sujetos de la investigación" a "entrevistados" o "participantes" demuestra la pretensión de hacer una investigación "con la gente" más que "sobre la gente".

\section{Casos de estudio}

Los profesionales que conforman el perfil de casos de estudio son Ignacio Escolar, Mikel López-Iturriaga y José-Antonio Pérez. Han sido escogidos por dos razones:

- son profesionales de éxito en España gracias a su trabajo en internet: Escolar en el análisis de la actualidad política, Iturriaga en periodismo especializado en gastronomía y Pérez en información de humor y divulgación de la ciencia;

- su influencia en la Red es ampliamente reconocida: en octubre de 2017 Ignacio Escolar tenía 770.000 seguidores en Twitter; Mikel López-Iturriaga 248.000 y José-Antonio Pérez 122.000. El índice Klout ${ }^{1}$ es de 74 puntos en Ignacio Escolar, 65 puntos en Mikel López-Iturriaga y 65 en José-Antonio Pérez.

\section{Empleadores}

Mikel Iturralde, Iñigo Kortabitarte, Javier Moltó, Gumersindo Lafuente e Ignacio Escolar conforman el perfil de empleadores. Se han seleccionado porque:
- Gumersindo Lafuente dirigió de 2010 a 2012 la transformación digital del diario El país, al que llegó tras su experiencia al frente del medio digital Soitu.es, que él mismo fundó. Durante seis años (2000 a 2006) dirigió la versión online del diario El mundo.

- Mikel Iturralde ha sido responsable del área digital del diario El correo hasta 2014, para pasar a dirigir la presencia en redes sociales del periódico líder en el País Vasco.

- Iñigo Kortabitarte es el director de innovación y desarrollo en Grupo El Diario Vasco Multimedia. El correo y el Diario vasco pertenecen a Vocento, uno de los grupos de comunicación más relevantes de España, formado por más de 100 empresas.

- Ignacio Escolar cumple la doble condición de caso de estudio y empleador porque se encarga personalmente de las contrataciones.

- Javier Moltó es un periodista especializado en automóvil. Es el responsable y creador de la revista digital del automóvil Km77.com.

Pese a su indiscutida relevancia, la reputación online tiene mayor influencia en los medios digitales que en los nacidos con el papel

\section{Expertos}

Por último, en el perfil de expertos se encuentran Silvia Cobo, Pedro de Alzaga, José Cervera, Rosalía Lloret y Fernando García-Mongay.

- Silvia Cobo es periodista y bloguera. Escribe sobre medios de manera independiente y es autora del libro Internet para periodistas (Cobo, 2012). Es responsable de redes sociales de El periódico de Catalunya.

- José Cervera fundó en 1999 Baquía.com, una web dedicada a la nueva economía. Más tarde montó la primera web del diario gratuito 20 minutos, con la primera licencia copyleft en un medio informativo comercial. Fundó el primer blog de la prensa española en 2004, Retiario, un blog de ciencia y tecnología que se albergó en El mundo, 20 minutos y que actualmente se encuentra en rtve.es.

- Pedro de Alzaga es subdirector de Cuartopoder.com, el primer periódico de blogs en castellano. Ha trabajado como editor en las versiones digitales de El país y El mundo.

- Fernando García-Mongay es el director general de Administración Electrónica y Sociedad de la Información del Gobierno de Aragón. Ha dirigido en todas sus ediciones el Congreso nacional de periodismo digital que se celebra en Huesca.

- Rosalía Lloret es directora de relaciones institucionales en la Asociación Europea de Editores Online, y miembro del consejo del fondo The Digital Initiative News de Google. Ha sido directora general de desarrollo digital en Prisa Noticias entre 2011 y 2013, responsable de desarrollo digital de la empresa Unidad Editorial y directora de la división de medios interactivos de Radio Televisión Española.

Se confeccionaron tres tipos de cuestionarios -uno para cada perfil- semiestructurados y focalizados, con un orden de preguntas abiertas a partir de los objetivos y las hipó- 
tesis que se plantearon. La razón por la cual se diseñaron tres entrevistas fue la intención de obtener distintas perspectivas sobre los mismos temas. A cada entrevistado de cada perfil se le formularon las mismas preguntas para asegurar los resultados de la investigación y que fuesen susceptibles de comparación (Taylor; Bogdan, 1992, p. 101).

De las 12 entrevistas, 6 se realizaron de manera presencial y las otras 6 mediante Skype y teléfono con una duración media de una hora.

\section{Análisis}

\subsection{Marca personal y reputación online, el nuevo currículum}

Una marca personal y una buena reputación online son imprescindibles para el acceso al mercado laboral de un periodista y/o comunicador. Así lo defienden los 3 casos de estudio, y 8 de los 9 expertos y empleadores consultados. Desde la perspectiva de estos últimos, se destaca el valor de internet como ventana para conocer a posibles candidatos al puesto de trabajo. Gumersindo Lafuente habla de "rastro digital", mientras Kortabitarte e Iturralde insisten en la relevancia de la "reputación", amplificada por internet.

Esa importancia no tiene siempre una traslación práctica. Los empleadores de medios impresos entrevistados no toman como referencia de contratación la marca personal, algo que sin embargo sí tiene muy presente un medio nativo digital como Eldiario.es, tal y como destaca Escolar, que prefiere denominarla "firma digital". Parece por tanto que pese a su indiscutida relevancia, la reputación online tiene mayor eco e influencia en los medios digitales que en los nacidos con el papel.

Al igual que en los casos de estudio, empleadores y expertos coinciden en subrayar la importancia del blog y Twitter como herramientas para la creación de la marca personal de un periodista, a la que catalogan como fundamental para poder trabajar. Dentro de ese binomio, es precisamente Twitter el medio al que Escolar y Pérez conceden mayor relevancia. Para Pérez son "pocos" los que utilizan el blog, y Escolar lo expresa de otra forma:

"La gente ya no visita mi blog todos los días, sino que me lee en Twitter todos los días".

Sin embargo, expertos como Pedro de Alzaga y Rosalía Lloret defienden la vigencia del blog. Para Lloret:

"Siendo periodista no sólo hay que usar Twitter, sino también el blog. Es muy relevante porque permite ver nuestro trabajo".
La utilidad de Facebook, sin embargo, genera más reticencias entre los tres perfiles entrevistados para esta investigación.

"Para crear marca personal la red es Twitter, no las redes en general, sino Twitter y los blogs" (Fernando García-Mongay).

"Facebook ha estado desde el principio basada en relaciones de confianza porque tú no añades a gente que no conoces; la gente lo usa con sus amigos, y no todos tus amigos son periodistas. Yo las cosas de trabajo las dejo en el Twitter" (Cobo).

Sin embargo, no se puede dejar de lado esta red social. Escolar explica:

"En las estadísticas de Eldiario.es, Facebook nos manda un $30 \%$ o un $40 \%$ más de audiencia que Twitter. Lo que pasa es que en Twitter tú puedes actuar, en Facebook no. 
Facebook genera más tráfico, pero es mucho más difícil que tú influyas en ese tráfico. Lo que sí puedes generar es una reacción en cadena en Twitter que provocará una reacción en cadena en Facebook, pero la de Facebook no se provocará en Twitter".

Pese a su indiscutida relevancia, su utilidad como medio de creación de marca personal, reputación y obtención de empleo queda a juicio de los participantes en este estudio en un segundo plano.

Sobre la posible sustitución del tradicional currículum vitae por el uso de Twitter y blog de manera profesional, los tres comunicadores han respondido de manera afirmativa, ya que opinan de manera conjunta que internet ha facilitado la capacidad de demostrar lo que uno sabe hacer.

"A mí nunca me han pedido el currículum, jamás", asegura José-A. Pérez.

Acerca de los requisitos que se demandan en los nuevos profesionales, los nueve empleadores y expertos coinciden en un punto: deben contar con las aptitudes más tradicionales, es decir, curiosidad, trabajo y esfuerzo, además de una buena calidad de redacción. Los expertos, en particular Alzaga, Lloret y García-Mongay añaden los conocimientos vinculados con la tecnología y la programación para que el periodista logre un mejor aprovechamiento de su labor en la Red.

"Los periodistas piensan que el lenguaje html es para los informáticos, pero dominar de forma básica esta herramienta te da una independencia fabulosa" (Alzaga).

\subsection{Blog y Twitter, fundamentales con funciones diferenciadas}

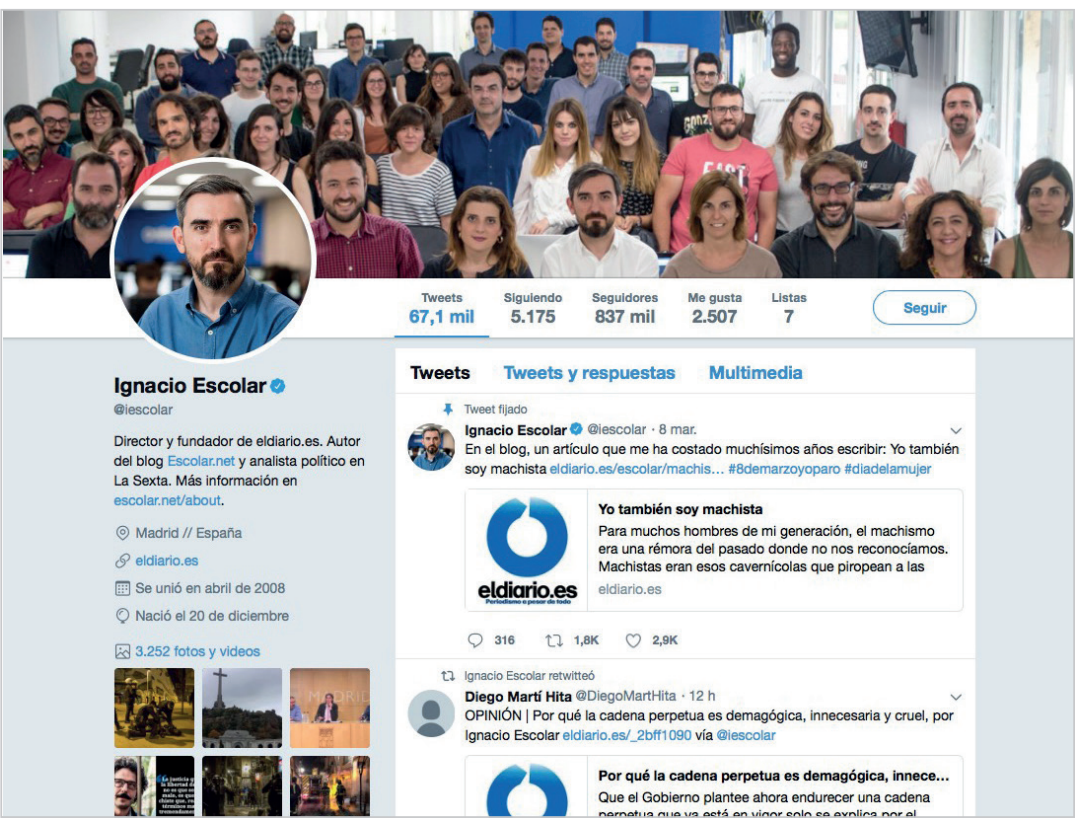

https://twitter.com/iescolar?lang=es

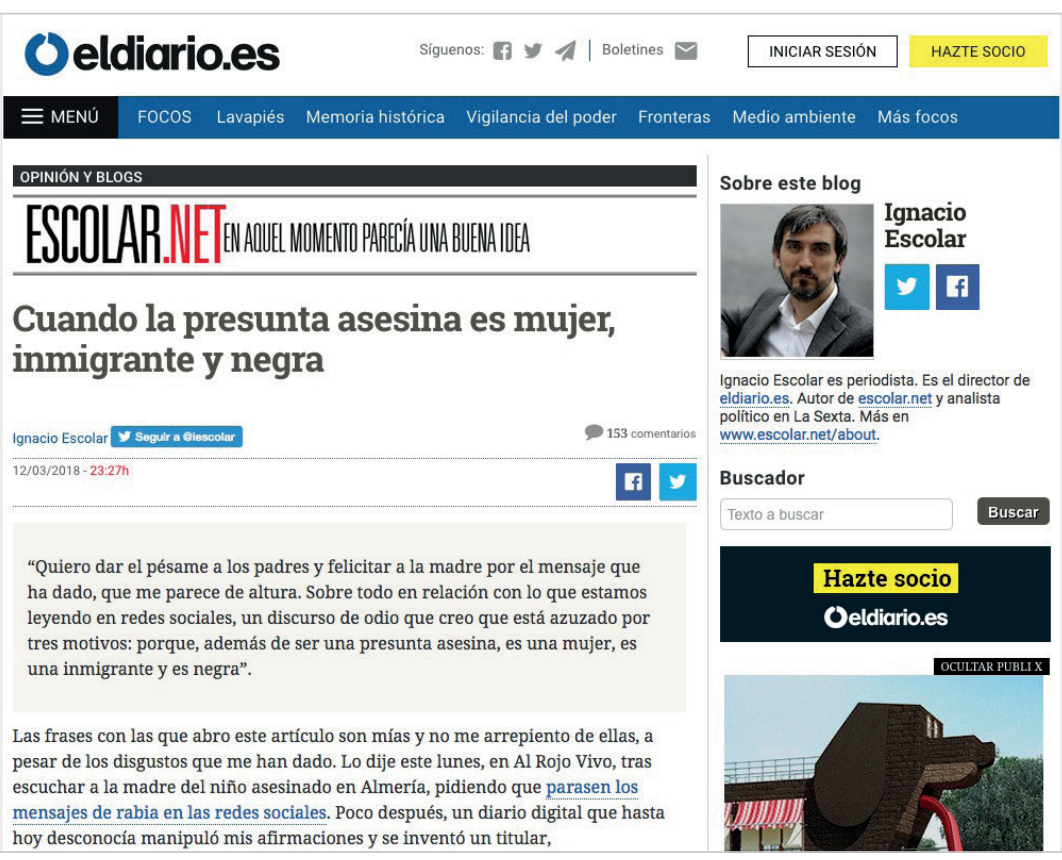

Los 3 casos de estudio, así como 8 de los 9 expertos y empleadores, defienden que el blog y Twitter son fundamentales para los profesionales de la comunicación. López-Iturriaga es claro:

"En mi caso, han servido para darme a conocer y para construirme un nombre".

Su éxito con su blog y su cuenta de Twitter permitió que un medio de referencia como El país lo fichara.

Similar es el caso de José-Antonio Pérez, quien indica:

"Estoy en Cadena Ser por el blog. He firmado un contrato con Planeta para hacer una novela, y me han llamado gracias a Mimesacojea.com".

Escolar realiza una descripción similar:

“Cuando empecé a hacer mi blog en 2003, era un periodista especializado en tecnología. (...) Empecé a escribir de política en mis ratos libres en el blog y eso provocó que mis asuntos laborales pasaran a ser de política".

\section{Twitter asume una gran relevancia como instrumento de promoción de conteni- dos para los que el blog es una platafor- ma idónea}

En cuanto a las funciones que ejercen Twitter y blog, los profesionales entrevistados coinciden: Twitter asume una gran relevancia como instrumento de promoción de contenidos 
para los que el blog es una plataforma idónea. Así lo confirman Pérez, Iturriaga y Escolar. Este último subraya:

"Para nosotros, cuando se publica una información, empieza el trabajo, no termina"

Así lo secundan el resto de personas entrevistadas. La experta y bloguera Silvia Cobo explica:

"Todos los blogueros están en Twitter. La blogosfera hace años que se mudó a Twitter. El día que no tuiteo o hago post tengo mucha menos visibilidad".

"Twitter es la red por excelencia de los periodistas. Si quieres trabajar de periodista ahora probablemente sea imprescindible estar en Twitter" (José Cervera).

Todos los entrevistados insisten en la necesidad de limitar la carga promocional para no perder la confianza de usuarios que pueden ser además futuros clientes, opinión compartida por los empleadores y expertos entrevistados. José-Antonio Pérez lo explica de este modo:

"A raíz de charlar a través de Twitter nos hemos acabando conociendo en la vida real, y hablo de periodistas y directores de medios".

Las funciones a las que destinan su trabajo en Twitter no acaban con la promoción propia. También lo utilizan para:

- opinar sobre temas y contenidos publicados en otros medios;

- obtener una mayor información;

- estar en contacto con los usuarios.

"Twitter también permite compartir la información, compartir fuentes y dialogar, esto último algo que no hemos hecho nunca en la prensa" (Alzaga).

\subsection{Blog y Twitter profesionales, no personales}

Una de las cuestiones que genera controversia entre expertos y profesionales es la conveniencia o no de mezclar asuntos personales y profesionales en las cuentas y perfiles de periodistas. Los tres entrevistados coinciden: adoptar posturas personales y profesionales en una red social puede ser peligroso.

"Cuando tienes muchos seguidores no puedes hacerlo, o no deberías hacerlo" (Escolar).

"Yo lo tengo completamente separado" (Pérez).

“A mí me parece una mezcla peligro- sa si no la haces bien porque puede resultar muy cansina (...). Jamás hablo de mi vida personal en Twitter" (López-Iturriaga).

En consonancia con lo expuesto por los casos de estudio, tanto empleadores como expertos reclaman moderación y prudencia. Según José Cervera:

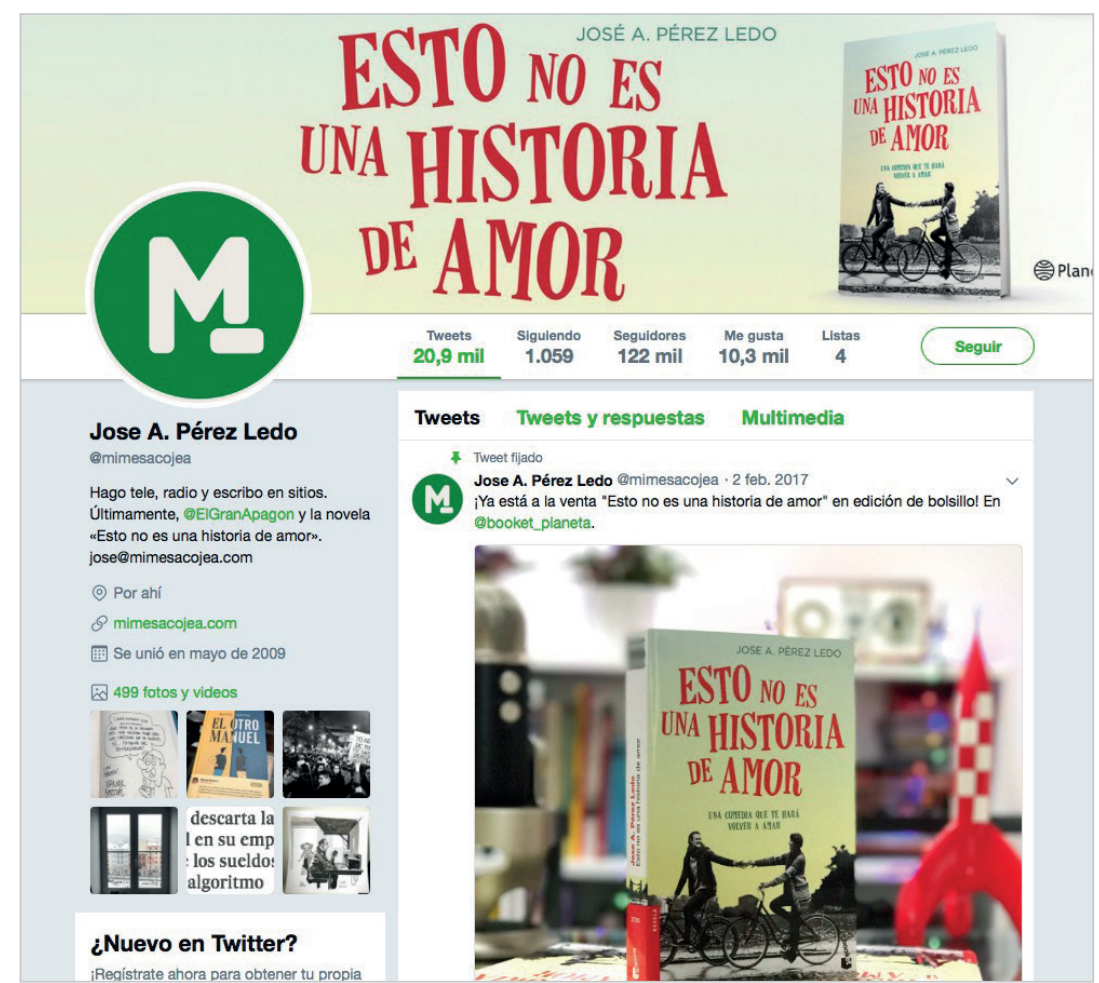

https://twitter.com/mimesacojea?lang=es
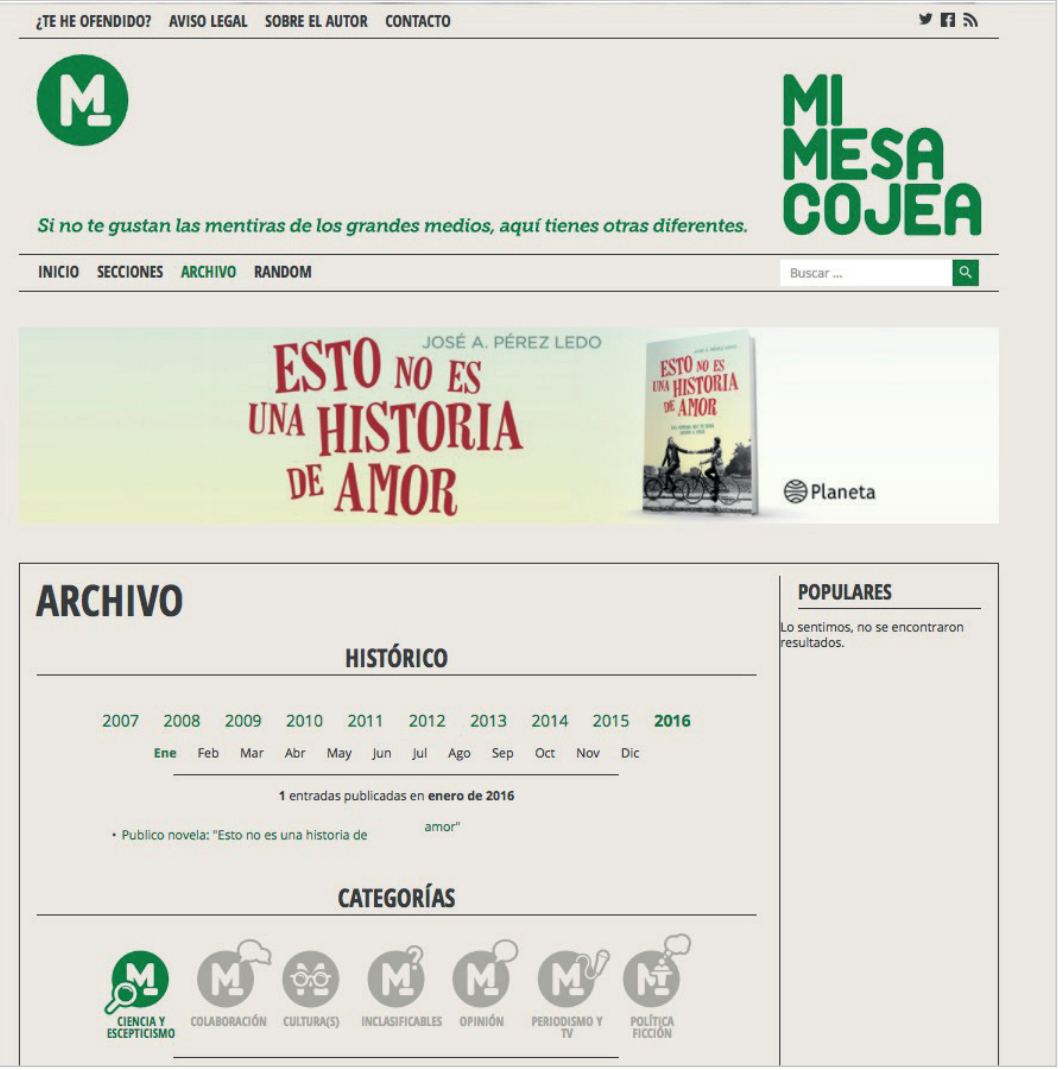

http://mimesacojea.com 
"En general, mezclar las dos cosas es complicado, por una cuestión de control y poder",

y utiliza como ejemplo los despidos vividos en Estados Unidos por los comentarios de periodistas en sus blogs y en sus canales de Twitter.

Iñigo Kortabitarte argumenta:

"Las redes sociales son una simbiosis entre el medio y el periodista que trabaja para ese medio, y esto tanto los medios como los periodistas lo deben entender".

"Puedes tener muchísimos seguidores en Twitter porque tu nombre o tu cara son conocidos, pero no ser influyente" (Mikel López-Iturriaga)

\subsection{Seguidores y medición}

Los tres casos de estudio coinciden en que el número de seguidores no es ni la única ni la mejor forma de medir la influencia y la marca personal en internet:

"Puedes tener muchísimos seguidores en Twitter porque tu nombre o tu cara son conocidos, pero no ser influyente" (Iturriaga).

"Twitter en realidad son universos paralelos (...) Al final, un tuit funciona como una reacción nuclear. Por tanto, el volumen importa, pero importa más dónde lo lances" (Escolar).

Los expertos y empleadores entrevistados también defienden que calidad y no cantidad, tanto en contenido como en influencia, son los factores clave:

"Durante mucho tiempo se ha valorado muchísimo la cantidad; creo que entramos en una fase mucho más madura en la cual empezamos a valorar incluso más la calidad" (Kortabitarte).

"Para mí sigue siendo más importante quién me sigue" (Cobo).

"Lo razonable no es tener 100.000 seguidores o $10 \mathrm{mi}$ llones de seguidores, lo razonable es tener influencia" (Cervera).

"Hay que establecer una ecuación entre calidad y relevancia" (Gumersindo Lafuente).

Cuestionados sobre la importancia que tiene la analítica web y el posicionamiento en Google en su uso de blog y Twitter, los tres tienen claro que lo más importante es que el contenido sea bueno, aunque difieren en algunos puntos. Para Ignacio Escolar, como director de Eldiario.es, esta medición tiene toda la importancia:

"Si no tienes información no tienes estrategia, necesitas saber exactamente lo que está pasando".

Por el contrario, Iturriaga asegura que le ha ido muy bien dejando a un lado el posicionamiento y José A. Pérez confiesa que no le hace caso ni a Google ni a la analítica:

"Cuando creé el blog sí que lo miraba porque pasas de que no te lea nadie a que te empiece a leer muchísima gente, pero hace tiempo que no me preocupa".

\section{Conclusiones}

Pese a ser un campo de estudio aún en ciernes y con referencias temporales muy recientes y limitadas a Estados Unidos (Molyneux; Holton, 2015; Molyneux, 2015), la relación entre marca personal, periodismo y redes sociales atrae la atención de un número creciente de investigaciones que analizan esta realidad en otros ámbitos (Brems et al., 2017; López-Meri; Casero-Ripollés, 2017; Hanusch; Bruns, 2017). Una de las razones la hallamos en los resultados de la presente investigación. Pese a las limitaciones por el reducido número de casos seleccionados y por el carácter netamente cualitativo de su análisis, ofrecen un bosquejo de una realidad que precisaría continuidad y profundización desde una perspectiva tanto cualitativa como cuantitativa.

Los 12 profesionales y expertos entrevistados, todos ellos con una dilatada y relevante trayectoria en el ejercicio del periodismo en internet, coinciden en que un periodista/comunicador con una sólida marca personal y una firma reconocida en internet tiene más posibilidades de encontrar trabajo que un profesional que carezca de ellas, afirmación que se alinea con los resultados de otros estudios similares (Hanusch; Bruns, 2017).

\section{Un periodista/comunicador con una só- lida marca personal y una firma recono- cida en internet tiene más posibilidades de encontrar trabajo}

De las respuestas de los profesionales "empleadores" se deduce que también es importante tener una buena reputación. Constatan así la relevancia que las redes sociales pueden tener en los periodistas, en especial en los freelancers, para ser emprendedores con su propia marca y entrar en contacto con potenciales clientes (Brems et al., 2017). es lo que ha ocurrido en los tres casos de estudio. No obstante, de los cinco empleadores sólo Ignacio Escolar subraya la importancia que tiene en su medio la actividad en la Web a la hora de contratar. La relevancia concedida casa con los resultados de estudios similares, en los que periodistas especializados señalan con claridad que no pueden atisbar un futuro halagüeño para los profesionales de la información que obvien una labor efectiva de creación de marca (Molyneux; Holton, 2015).

Y en esa labor, la promoción del propio trabajo representa un elemento clave a juicio de los 3 casos de estudio. A este respecto cobra especial relevancia Twitter. Coinciden con los resultados obtenidos en investigaciones precedentes, en las que se comprueba que los propios periodistas se ven como "publiperiodistas", es decir, publicistas de su propia marca y de la organización para la que trabajan (Molyneux; Holton, 2015, p. 233). Sin embargo, y he ahí una prometedora línea de investigación que el presente artículo bosqueja, la labor de creación de marca en el uso periodístico de Twitter es, a diferencia de lo que sucede con la responsabilidad y la recopilación de noticias, un campo de estudio poco explorado (Hanusch; Bruns, 2017). 
Para el logro de una promoción real, los periodistas y expertos entrevistados alertan de la necesidad de que esa labor sea comedida para no perder así credibilidad ni atracción entre sus seguidores. Esta prevención incide en la línea recogida en estudios previos de incluir como elemento reforzador de la marca personal la promoción de terceros (Molyneux, 2015) y, con ello, de la transparencia, credibilidad e integridad.

Twitter y blog son, en opinión de expertos, empleadores y profesionales, los medios más útiles para la creación de una marca personal y una posterior reputación online. Ninguno de ellos incluye a Facebook, pese a ser la red más extendida y que aporta mayor volumen de tráfico a los medios, por considerarla más rígida y menos manejable. Los tres casos estudiados coinciden en que el reflejo de su trabajo en su blog y Twitter les ha permitido alcanzar la posición que ocupan. Escolar, Pérez y López-Iturriaga señalan que el reconocimiento les ha venido dado gracias, de forma particular, a su blog, potenciado con la labor complementaria en Twitter. Esto les ha ayudado en la obtención de diferentes trabajos, lo que refleja la utilidad y eficacia de estas herramientas para los profesionales de la comunicación. Más aún si se tiene en consideración la pujanza de la figura del periodista emprendedor (Briggs, 2012). Es un argumento más para interiorizar la labor de marca personal como una parte integral del trabajo periodístico pese a la ausencia de directrices sobre cómo construirla (Molyneux; Holton, 2015). Se deduce de ello la necesidad de profundizar en posteriores estudios en el establecimiento de pautas y guías para el desarrollo de la marca personal en el mundo periodístico.

\section{Twitter y blog son, en opinión de exper- tos, empleadores y profesionales, los medios más útiles para la creación de una marca personal y una posterior re- putación online}

En todo caso, y esta afirmación se repite entre los casos de estudio y el resto de perfiles entrevistados, la marca personal no depende del número de seguidores que se posee en Twitter ni del número de visitas del blog, sino de la calidad de los contenidos que se difundan y de quiénes sean sus seguidores. Las doce personas entrevistadas en esta investigación señalan unánimemente que el número de seguidores no representa una variante clave.

La mayoría de los entrevistados defienden que la combinación de aspectos personales y profesionales en un perfil de red social es desaconsejable. De hecho, ninguno de los tres casos de estudio lo hace. Esta coincidencia contrasta con los resultados obtenidos hasta ahora en los escasos estudios que han abordado la construcción de la marca personal en las redes sociales, en particular en Twitter, que destacan la necesidad de fusionar una identidad profesional y personal (Molyneux; Holton, 2015, Brems et al., 2017, Hanusch; Bruns, 2017) para lograr una transparencia clave en la presentación y desarrollo de la marca personal en redes sociales.

El perfil de periodista que se desprende de esta investigación es el de un profesional con dominio del entorno online, con amplio control de las aplicaciones que éste ofrece y capaz de crear su marca personal para lograr ser influyente en el nicho de mercado al que van dirigidas sus publicaciones. Se deduce del análisis de las respuestas de empleadores y expertos, y de los ejemplos de casos de estudio, que el profesional debe además las aptitudes tradicionales del periodismo, así como disponer de fuentes de calidad y habilidad para buscar información de interés. Esta visión coincide con los resultados de investigaciones centradas en el uso periodístico de blogs y Twitter (Singer, 2005; Lasorsa; Lewis; Holton, 2012), que recalcan que estas herramientas pasan a ser un componente e incluso un refuerzo de las normas y prácticas tradicionales periodísticas.

\section{Escolar, El comidista y Mi mesa cojea coinciden en que no es conveniente mezclar asuntos personales y profesio- nales en las cuentas de redes sociales}

\section{Nota}

1. El índice Klout mide la influencia de una persona en las redes sociales. Se considera influencer o persona influyente aquella que obtiene una puntuación mayor a 60 .

\section{Agradecimientos}

Resultado del proyecto CSO2014-46196-R “Aplicación de la convergencia hipermedia en la comunicación corporativa: Sala de Comunicación Abierta (SCA)" del Ministerio de Economía y Competitividad (Mineco) de España.

\section{Referencias}

APM (2016). Informe anual de la profesión periodística. Asociación de la Prensa de Madrid.

Arrabal-Sánchez, Gabriel; De-Aguilera-Moyano, Miguel (2016). "Comunicar en 140 caracteres. Cómo usan Twitter los comunicadores en España". Comunicar, n. 46, pp. 9-17. https://doi.org/10.3916/C46-2016-01

Bakker, Piet (2014). "Mr. Gates returns. Curation, community management and other new roles for journalists". Journalism studies, v. 15, n. 5, pp. 596-606.

https://doi.org/10.1080/1461670X.2014.901783

Banister, Peter; Burman, Erica; Parker, Ian; Taylor, Maye; Tindall, Carol (2004). Métodos cualitativos en Psicología: una guía para la investigación. Guadalajara: Universidad de Guadalajara. ISBN: 9789687846408

http://newpsi.bvs-psi.org.br/ebooks2010/pt/Acervo_files/ MetodosCualitativos-completo.pdf

Brems, Cara; Temmerman, Martina; Graham, Todd; Broersma, Marcel (2017). "Personal branding on Twitter: How employed and freelance journalists stage themselves on social media". Digital journalism, v. 5, n. 4, pp. 443-459. https://doi.org/10.1080/21670811.2016.1176534

Briggs, Mark (2012). Entreprenurial journalism: How to build what's next for news. Los Angeles: Sage. ISBN: 9781 608714209 
Campos-Freire, Francisco; Rúas-Araújo, José; López-García, Xosé; Martínez-Fernández, Valentín-Alejandro (2016). "Impacto de las redes sociales en el periodismo". El profesional de la información, v. 25, n. 3, pp. 449-457. https://doi.org/10.3145/epi.2016.may.15

Cobo, Silvia (2012). Internet para periodistas. Kit de supervivencia para la era digital. Barcelona: Editorial UOC. ISBN: 9788497889889

Hanusch, Folker; Bruns, Axel (2017). "Journalistic branding on Twitter. A representative study of Australian journalists' profile descriptions". Digital journalism, v. 5, n. 1, pp. 26-43. https://eprints.qut.edu.au/94315

https://doi.org/10.1080/21670811.2016.1152161

Hedman, Ulrika; Djerf-Pierre, Monika (2013). "The social journalist". Digital journalism, v. 1, n. 3, pp. 368-385. https://doi.org/doi:10.1080/21670811.2013.776804

Hermida, Alfred (2010). "Twittering the news. The emergence of ambient journalism". Journalism practice, v. 4, n. 3, pp. 297-308.

https://papers.ssrn.com/sol3/papers.cfm?abstract id $=1732598$ https://doi.org/10.1080/17512781003640703

Hermida, Alfred (2013). “\#Journalism: Reconfiguring journalism research about Twitter, one tweet at a time". Digital journalism, v. 1, n. 3, pp. 295-313.

https://doi.org/10.1080/21670811.2013.808456

Jarvis, Jeff (2014). Geeks bearing gifts: Imagining new futures for news. New York: Cuny Journalism Press. ISBN: 978 1939293749

Lasorsa, Dominic; Lewis, Seth; Holton, Avery (2012). “Normalizing Twitter. Journalism practice in an emerging communication space". Journalism studies, v. 13, n. 1, pp. 19-36. https://conservancy.umn.edu/handle/11299/123293 https://doi.org/10.1080/1461670X.2011.571825

López-Meri, Amparo (2015). "El impacto de Twitter en el periodismo: un estado de la cuestión". Revista de la Asociación Española de Investigación de la Comunicación, v. 2, pp. 34-41. http://repositori.uji.es/xmlui/handle/10234/165677

López-Meri, Amparo; Casero-Ripollés, Andreu (2016). “EI debate de la actualidad periodística española en Twitter: Del corporativismo de periodistas y políticos al activismo ciudadano". Observatorio (OBS*), v. 10, n. 3, pp. 56-79. http://obs.obercom.pt/index.php/obs/article/view/994

López-Meri, Amparo; Casero-Ripollés, Andreu (2017). “Las estrategias de los periodistas para la construcción de marca personal en Twitter: posicionamiento, curación de contenidos, personalización y especialización". Revista mediterránea de comunicación, v. 8, n. 1, p. 59-73.

https://doi.org/10.14198/MEDCOM2017.8.1.5

Molyneux, Logan (2015). "What journalists retweet: Opinion, humor, and brand development on Twitter". Journal- ism, v. 16, pp. 920-935.

https://goo.gl/qGbsWa

https://doi.org/10.1177/1464884914550135

Molyneux, Logan; Holton, Avery (2015). "Branding (health) journalism: Perceptions, practices, and emerging norms". Digital journalism, v. 3, n. 2, pp. 225-242.

https://doi.org/10.1080/21670811.2014.906927

Noguera-Vivo, José-Manuel (2013). "How open are journalists on Twitter? Trends towards the end-user journalism". Communication \& society, v. 26, n. 1, pp. 93-114.

https://www.unav.es/fcom/communication-society/es/ articulo.php?art_id $=438$

Palomo, Bella (2013). "Claves de la implantación y la expansión del perfil social del periodista". Comunicación y medios, v. 28 , pp. $113-129$.

https://doi.org/10.5354/0716-3991.2013.27403

Palomo, Bella; Palau-Sampio, Dolors (2016). “El periodista adaptativo. Consultores y directores de innovación analizan las cualidades del profesional de la comunicación". El profesional de la información, v. 25, n. 2, pp. 188-195. https://doi.org/10.3145/epi.2016.mar.05

Pérez-Ortega, Andrés (2012). "Branding personal. El ejemplo de los periodistas". Marcapropia.net, 11 diciembre. https://www.andresperezortega.com/2012/12/brandingpersonal-el-ejemplo-de-los-periodistas.html

Picard, Robert G. (2014). "Twilight or new dawn of journalism?". Digital journalism, v. 2, n. 2, pp. 273-283.

https://goo.gl/wgoNdr https://doi.org/10.1080/21670811.2014.895531

Picard, Robert G. (2015). Journalists' perceptions of the future of journalistic work. Reuters Institute for the Study of Journalism. University of Oxford. https://goo.gl/eSL3CV

Rodríguez-Rubial, Antonio; García-López, Javier (2013). "Uso y funciones de Twitter en periodistas españoles". Estudios sobre el mensaje periodístico, v. 19, pp. 963-969. https://doi.org/10.5209/rev_ESMP.2013.v19.42181

Singer, Jane B. (2005). "The political j-blogger: 'Normalizing' a new media form to fit old norms and practices". Journalism, v. 6, n. 2, pp. 173-198.

http://openaccess.city.ac.uk/3463/

https://doi.org/10.1177/1464884905051009

Swasey, Alecia (2017). "I studied how journalists used Twitter for two years. Here's what I learned". Poynter.org, 22 March.

https://www.poynter.org/2017/i-studied-how-journalistsused-twitter-for-two-years-heres-what-i-learned/453162

Taylor, Steve J.; Bogdan, Robert (1992). Introducción a los métodos cualitativos de investigación. Paidós: Barcelona. ISBN: 8475098169 